Article

\title{
Vectorizing Pro-Insecticide: Influence of Linker Length on Insecticidal Activity and Phloem Mobility of New Tralopyril Derivatives
}

\author{
Tian Xing Li ${ }^{1}$, Yao Chen ${ }^{2}$, Hui Fang Liu ${ }^{2}$, Chi Yu Ma ${ }^{2}$ and Wen Yang ${ }^{2, *}$ \\ 1 College of Tea Science, Guizhou University, Guiyang 550025, China; 18586822040@163.com \\ 2 Guizhou Tea Research Institute, Guizhou Academy of Agricultural Sciences, Guiyang 550006, China; \\ cheny0221@163.com (Y.C.); 18300865026@163.com (H.F.L.); chiyuma81@163.com (C.Y.M.) \\ * Correspondence: yangwen3409@126.com
}

Citation: Li, T.X.; Chen, Y.; Liu, H.F.; Ma, C.Y.; Yang, W. Vectorizing Pro-Insecticide: Influence of Linker Length on Insecticidal Activity and Phloem Mobility of New Tralopyril Derivatives. Molecules 2021, 26, 4570. https://doi.org/10.3390/

molecules 26154570

Academic Editor: Josphat Matasyoh

Received: 21 June 2021

Accepted: 23 July 2021

Published: 28 July 2021

Publisher's Note: MDPI stays neutral with regard to jurisdictional claims in published maps and institutional affiliations.

Copyright: () 2021 by the authors. Licensee MDPI, Basel, Switzerland. This article is an open access article distributed under the terms and conditions of the Creative Commons Attribution (CC BY) license (https:// creativecommons.org/licenses/by/ $4.0 /)$.

\begin{abstract}
To improve the proinsecticidal activity and phloem mobility of amino acid-tralopyril conjugates further, nine conjugates were designed and synthesized by introducing glutamic acid to tralopyril, and the length of the linker between glutamic acid and tralopyril ranged from 2 atoms to 10 atoms. The results of insecticidal activity against the third-instar larvae of $P$. xylostella showed that conjugates $\mathbf{4 2}, \mathbf{4 3}, \mathbf{4 4}$,and 45 (straight-chain containing 2-5 atoms) exhibited good insecticidal activity, and their $\mathrm{LC}_{50}$ values were $0.2397 \pm 0.0366,0.4413 \pm 0.0647,0.4400 \pm 0.0624$, and $0.4602 \pm 0.0655 \mathrm{mM}$, respectively. The concentrations of conjugates $43-45$ were higher than that of conjugate $\mathbf{4 2}$ in the phloem sap at $2 \mathrm{~h}$, and conjugate $\mathbf{4 3}$ showed the highest concentration. The introduction of glutamic acid can improve phloem mobility. The in vivo metabolism of conjugates 42 and 43 was investigated in P.xylostella, and the parent compound tralopyril was detected at concentrations of 0.5950 and $0.3172 \mathrm{nmol} / \mathrm{kg}$, respectively. According to the above results, conjugates 42 and $\mathbf{4 3}$ were potential phloem mobile pro-insecticide candidates.
\end{abstract}

Keywords: insecticide conjugate; glutamic acid; tralopyril; chlorfenapyr; phloem mobility

\section{Introduction}

The phloem systemicity of pesticides is an important characteristic for the expression of their biological activities [1,2]. Insecticides with phloem mobility are preferred for controlling pests hidden on unpredictable nonexposed plant parts, such as growing tips, roots, inside leaf deformations, and galls [3]. However, few existing insecticides have good phloem mobility. Currently, only spirotetramat is an ambimobile systemic (phloem and xylem systemic) insecticide in plants, which can be used to control hidden and soil living sucking pests, such as aphids and whiteflies [3].

The carrier-mediated strategy has been used to develop novel phloem mobile insecticides [1]. Several attempts have been made to achieve phloem-mobile insecticides by introducing a carboxyl group, amino acid, or sugar to the parent compounds of existing non-phloem-mobile pesticides [2,4,5]. Pioneering work was conducted to achieve phloem-mobile pronematicide by introducing glucuronic acid moiety to oxamyl [4]. One vectorization strategy of agrochemicals has been established by introducing carboxyl group, amino acid, and sugar to phenylpyrrole fungicide [6-8]. Plant amino acid and sugar transporters have been reported to interact with pesticide conjugates and enhance their phloem translocation $[9,10]$.

Tralopyril (Figure 1) is a biocide optimized from dioxapyrrolomycin that shows excellent insecticidal activity [11,12]. However, this compound exhibits phytotoxic effects. Chlorfenapyr is the first commercial proinsecticide developed by introducing ethoxymethyl group into the pyrrole $\mathrm{N}$ of tralopyril [11-14]. Tralopyril is a nonsystemic insecticide without phloem mobility. To address the problems of systemicity and phytotoxicity, a strategy 
of vectorizing the pro-insecticide of tralopyril was proposed in our research group. The six new conjugates were designed and synthesized by introducing glucose, methyl glucuronate, and glucuronic acid moieties into tralopyril. The phloem mobility results demonstrated that our strategy can improve the phloem mobility of tralopyril $[15,16]$.

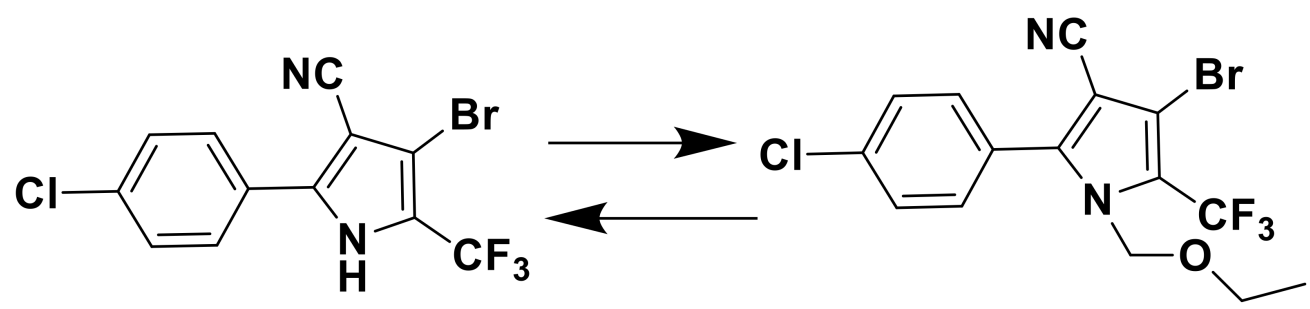

Figure 1. Chemical structure of tralopyril and chlorfenapyr.

Our previous results demonstrated that introducing glutamic acid moieties can improve the phloem mobility of tralopyril [16]. However, the structure-activity relationship of tralopyril conjugates has not yet been investigated, especially for the linker structure. In previous studies, the linker structure between the nutrient promoiety and active ingredient influence the bioactivity and phloem mobility of the conjugates [8,17]. Hence, the aim of the present study is to elucidate the structure-activity relationship for the linker between amino acid promoiety and tralopyril. (I) Nine tralopyril conjugates containing glutamic acid with different linker lengths were synthesized. (II) The insecticidal activity of the conjugates against the third-instar larvae of $P$. xylostella was evaluated using a poison fodder method. (III) Phloem mobility test in castor bean seedlings was performed. (IV) The metabolism of conjugates $\mathbf{4 2}$ and $\mathbf{4 3}$ in P. xylostella was investigated. This study provides a fundamental understanding of the influence of different linker lengths on the insecticidal activity and phloem mobility of new tralopyril derivatives.

\section{Materials and Methods}

\subsection{Synthesis}

\subsubsection{General Information for Synthesis}

Solvents were of analytical grade and were dried according to the methods of the Purification of Laboratory Chemicals, Fifth Edition. Reagents were purchased from a commercial company. ${ }^{1} \mathrm{H}$ NMR and ${ }^{13} \mathrm{C}$ NMR spectra were obtained on a Varian INOVA400 instrument. Chemical shifts were expressed in $\delta(\mathrm{ppm})$ values, with TMS as an internal standard. Analytical thin-layer chromatography (TLC) was performed on silicagel GF254. Silica gel (100-200 and 200-300 mesh, Qingdao Marine Chemical Ltd., Qingdao, China) was used for column chromatography.

\subsubsection{Synthesis of Compound 4}

Synthesis of the title compound was accomplished according to a previously reported procedure [16]. $\mathrm{NaH}(60 \%$ in oil, $1 \mathrm{~g}, 25 \mathrm{mmol})$ was added to the solution of tralopyril $(3.5 \mathrm{~g}, 10 \mathrm{mmol})$ in a mixture solvent of dry THF $(30 \mathrm{~mL})$ and bromochloromethane $(12.94 \mathrm{~g}$, $100 \mathrm{mmol}, 5$ equiv). The mixture was then stirred at room temperature for $2 \mathrm{~h}$. Propargyl alcohol $(10 \mathrm{mmol})$ in THF $(10 \mathrm{~mL})$ was added dropwise and stirred at $65^{\circ} \mathrm{C}$ for $48 \mathrm{~h}$. The reaction mixture was quenched by adding ice water, and the resultant mixture was extracted with ethyl acetate $(20 \mathrm{~mL} \times 3)$. The combined organic layers were washed with aqueous sodium hydrogen carbonate and brine, dried with sodium sulfate, filtered, and evaporated in vacuo. The residues were purified by column chromatography to obtain the desired product 4 as a white solid with $63 \%$ yield. ${ }^{1} \mathrm{H}$ NMR $\left(400 \mathrm{MHz}, \mathrm{DMSO}-d_{6}\right) \delta$ $7.69(\mathrm{~m}, 4 \mathrm{H}, \mathrm{Ar}-\mathrm{H}), 5.38(\mathrm{~s}, 2 \mathrm{H}), 4.05(\mathrm{~d}, J=2.4 \mathrm{~Hz}, 2 \mathrm{H}), 3.37(\mathrm{t}, J=2.4 \mathrm{~Hz}, 1 \mathrm{H}) .{ }^{13} \mathrm{C}$ NMR $\left(100 \mathrm{MHz}, \mathrm{DMSO}-d_{6}\right) \delta 144.60,135.88,132.04 \times 2,129.32 \times 2,125.26,121.31,118.66,113.61$, $103.44,97.96,78.10,77.96,74.77,55.65$. API-ES $(m / z): 441.00[\mathrm{M}+\mathrm{Na}]^{+}$. 


\subsubsection{General Procedures for Compounds 14-22}

Synthesis of compounds 14-22 followed the procedure previously reported by $\mathrm{Lu}$ and Bittman [16,18]. In a $20 \mathrm{~mL}$ flask, compounds 5-13 (10 mmol), $\mathrm{NaN}_{3}(0.85 \mathrm{~g}, 13.2 \mathrm{mmol})$, and $\mathrm{n}-\mathrm{Bu}_{4} \mathrm{NBr}(80 \mathrm{mg}, 0.25 \mathrm{mmol})$ were mixed and stirred for $15 \mathrm{~h}$ at $110^{\circ} \mathrm{C}$. After the mixture had cooled, the product was taken up with $\mathrm{CH}_{2} \mathrm{Cl}_{2}(20 \mathrm{~mL}$ ), and the precipitate (consisting of $\mathrm{NaBr}$, unreacted $\mathrm{NaN}_{3}$, and phase-transfer catalyst) was removed by filtration. The salt was washed with $\mathrm{CH}_{2} \mathrm{Cl}_{2}(20 \mathrm{~mL})$. Evaporation of the solvents resulted in a yellow residue that was purified by distillation at $35^{\circ} \mathrm{C}$ to yield compounds 14-22 as a colorless liquid.

\subsubsection{General Procedures for Compounds 23-31}

Synthesis of the title compound was accomplished according to a previously reported procedure [16]. Compounds 23-31 were synthesized using this procedure. Tralopyril derivative 4 (10 $\mathrm{mmol})$ and crude azide 14-22 $(10 \mathrm{mmol})$ were dissolved in $20 \mathrm{~mL}$ DMF. The reaction was initiated by the addition of $\mathrm{CuSO}_{4} \cdot 5 \mathrm{H}_{2} \mathrm{O}(4 \mathrm{mmol})$ and sodium ascorbate $(8 \mathrm{mmol})$ in $5 \mathrm{~mL}$ distilled water. The mixture was stirred at $60{ }^{\circ} \mathrm{C}$ until TLC indicated the disappearance of the starting materials. The mixture was poured into distilled water $(20 \mathrm{~mL})$, and the product was extracted three times with ethyl acetate $(15 \mathrm{~mL} \times 3)$. The organic layer was dried with $\mathrm{Na}_{2} \mathrm{SO}_{4}$ and filtered. The solvent was removed under reduced pressure. The residues were purified by column chromatography to obtain the compounds 23-31.

Compound 23: ${ }^{1} \mathrm{H}$ NMR $\left(400 \mathrm{MHz}, \mathrm{DMSO}-d_{6}\right) \delta 7.89(\mathrm{~s}, 1 \mathrm{H}), 7.64 \sim 7.67(\mathrm{~m}, 4 \mathrm{H})$, $5.38(\mathrm{~s}, 2 \mathrm{H}), 5.02(\mathrm{~s}, 1 \mathrm{H}), 4.41(\mathrm{~s}, 2 \mathrm{H}), 4.36(\mathrm{t}, 2 \mathrm{H}, J=3.6 \mathrm{~Hz}), 3.74(\mathrm{~s}, 2 \mathrm{H}) .{ }^{13} \mathrm{C}$ NMR $\left(100 \mathrm{MHz}, \mathrm{DMSO}-d_{6}\right) \delta 144.35,141.77,135.75,131.80,129.16 \times 2,125.20,124.52 \times 2,120.77$, $119.05,113.74,103.13,97.82,75.28,61.30,59.72,52.02$. API-ES $(m / z): 528.3[\mathrm{M}+\mathrm{Na}]^{+}$.

Compound 24: ${ }^{1} \mathrm{H}$ NMR $\left(400 \mathrm{MHz}, \mathrm{DMSO}-d_{6}\right) \delta 7.91(\mathrm{~s}, 1 \mathrm{H}), 7.65(\mathrm{~m}, 4 \mathrm{H}), 5.37(\mathrm{~s}, 2 \mathrm{H})$, $5.02(\mathrm{~s}, 1 \mathrm{H}), 4.43(\mathrm{~s}, 2 \mathrm{H}), 4.38(\mathrm{t}, 2 \mathrm{H}, J=4.8 \mathrm{~Hz}), 3.39(\mathrm{t}, 2 \mathrm{H}, J=4.0 \mathrm{~Hz}), 1.90 \sim 1.95(\mathrm{~m}, 2 \mathrm{H}) .{ }^{13} \mathrm{C}$ NMR $\left(100 \mathrm{MHz}\right.$, DMSO- $\left.d_{6}\right) \delta 144.35,141.92,135.81,131.79,129.17 \times 2,125.20,124.13 \times 2$, 120.78, 118.99, 113.48, 103.17, 97.87, 75.23, 61.33, 57.33, 46.57 32.88. API-ES $(m / z)$ : $542.3[\mathrm{M}+\mathrm{Na}]^{+}$.

Compound 25: ${ }^{1} \mathrm{H}$ NMR $\left(400 \mathrm{MHz}\right.$, DMSO- $\left.d_{6}\right) \delta 7.91(\mathrm{~s}, 1 \mathrm{H}), 7.65(\mathrm{~m}, 4 \mathrm{H}), 5.37(\mathrm{~s}, 2 \mathrm{H})$, $5.34(\mathrm{~s}, 1 \mathrm{H}), 4.43(\mathrm{~d}, 2 \mathrm{H}, J=4.4 \mathrm{~Hz}), 4.34 \sim 4.36(\mathrm{~m}, 2 \mathrm{H}), 3.42(\mathrm{t}, 2 \mathrm{H}, J=4.0 \mathrm{~Hz}), 1.80 \sim 1.83(\mathrm{~m}, 2 \mathrm{H})$, 1.34 1.38 (m, 2H). ${ }^{13} \mathrm{C}$ NMR (100 MHz, DMSO- $\left.d_{6}\right) \delta 144.33,142.00,135.77,131.78,129.14 \times 2$, $125.19,124.03 \times 2,120.75,118.97,113.45,103.13,97.85,75.24,61.30,59.90,49.20,29.10,26.65$. API-ES $(m / z): 556.3[\mathrm{M}+\mathrm{Na}]^{+}$.

Compound 26: ${ }^{1} \mathrm{H}$ NMR $\left(400 \mathrm{MHz}\right.$, DMSO- $\left.d_{6}\right) \delta 7.90(\mathrm{~s}, 1 \mathrm{H}), 7.62 \sim 7.65(\mathrm{~m}, 4 \mathrm{H})$, $5.37(\mathrm{~s}, 2 \mathrm{H}), 4.48(\mathrm{~s}, 1 \mathrm{H}), 4.44(\mathrm{~s}, 2 \mathrm{H}), 4.30(\mathrm{t}, 2 \mathrm{H}, J=4.8 \mathrm{~Hz}), 3.38(\mathrm{t}, 2 \mathrm{H}, J=4.4 \mathrm{~Hz})$, $1.75 \sim 1.80(\mathrm{~m}, 2 \mathrm{H}), 1.42 \sim 1.46(\mathrm{~m}, 2 \mathrm{H}), 1.21 \sim 1.27(\mathrm{~m}, 2 \mathrm{H}) .{ }^{13} \mathrm{C}$ NMR $\left(100 \mathrm{MHz}\right.$, DMSO- $\left.d_{6}\right) \delta$ $144.33,141.89,135.79,131.78,129.15 \times 2,125.19,123.91 \times 2,120.76,118.98,113.46,103.13$, $97.86,75.18,61.32,60.38,49.32,31.71,29.58,22.40$. API-ES $(m / z): 570.2[\mathrm{M}+\mathrm{Na}]^{+}$.

Compound 27: ${ }^{1} \mathrm{H}$ NMR $\left(400 \mathrm{MHz}, \mathrm{DMSO}-d_{6}\right) \delta 7.91(\mathrm{~s}, 1 \mathrm{H}), 7.62 \sim 7.66(\mathrm{~m}, 4 \mathrm{H})$, $5.38(\mathrm{~s}, 2 \mathrm{H}), 4.47(\mathrm{~s}, 2 \mathrm{H}), 4.37(\mathrm{~s}, 1 \mathrm{H}), 4.31(\mathrm{t}, 2 \mathrm{H}, J=4.8 \mathrm{~Hz}), 3.39(\mathrm{t}, 2 \mathrm{H}, J=3.6 \mathrm{~Hz})$, $1.76 \sim 1.81(\mathrm{~m}, 2 \mathrm{H}), 1.38 \sim 1.43(\mathrm{~m}, 2 \mathrm{H}), 1.29 \sim 1.34(\mathrm{~m}, 2 \mathrm{H}), 1.19 \sim 1.24(\mathrm{~m}, 2 \mathrm{H}) .{ }^{13} \mathrm{C}$ NMR $\left(100 \mathrm{MHz}, \mathrm{DMSO}-d_{6}\right) \delta 144.32,141.93,135.87,131.73,129.14 \times 2,125.18,123.89 \times 2,120.78$, $118.99,113.41,103.11,97.95,75.14,61.36,60.60,49.31,32.25,29.76,25.69,24.87$. API-ES $(m / z): 584.3[\mathrm{M}+\mathrm{Na}]^{+}$.

Compound 28: ${ }^{1} \mathrm{H}$ NMR $\left(400 \mathrm{MHz}, \mathrm{DMSO}-d_{6}\right) \delta 7.90(\mathrm{~s}, 1 \mathrm{H}), 7.62 \sim 7.66(\mathrm{~m}, 4 \mathrm{H})$, $5.37(\mathrm{~s}, 2 \mathrm{H}), 4.46(\mathrm{~s}, 2 \mathrm{H}), 4.34(\mathrm{~s}, 1 \mathrm{H}), 4.30(\mathrm{t}, 2 \mathrm{H}, J=4.8 \mathrm{~Hz}), 3.38(\mathrm{t}, 2 \mathrm{H}, J=4.0 \mathrm{~Hz})$, 1.74 1.79 (m, 2H), 1.37 1.41 (m, 2H), 1.23 1.28 (m, 4H), 1.17 1.22 (m, 2H). ${ }^{13} \mathrm{C}$ NMR $\left(100 \mathrm{MHz}, \mathrm{DMSO}-d_{6}\right) \delta 144.30,141.89,135.81,131.74,129.13 \times 2,125.18,123.88 \times 2,120.76$, $118.97,113.41,103.11,97.89,75.14,61.32,60.64,49.27,32.34,29.65,28.23,25.79,25.27$. API-ES $(m / z): 598.3[\mathrm{M}+\mathrm{Na}]^{+}$.

Compound 29: ${ }^{1} \mathrm{H}$ NMR $\left(400 \mathrm{MHz}, \mathrm{DMSO}-d_{6}\right) \delta 7.91(\mathrm{~s}, 1 \mathrm{H}), 7.62 \sim 7.66(\mathrm{~m}, 4 \mathrm{H})$, $5.36(\mathrm{~s}, 2 \mathrm{H}), 4.46(\mathrm{~s}, 2 \mathrm{H}), 4.33(\mathrm{~s}, 1 \mathrm{H}), 4.30(\mathrm{t}, 2 \mathrm{H}, J=4.4 \mathrm{~Hz}), 3.38(\mathrm{t}, 2 \mathrm{H}, J=4.4 \mathrm{~Hz})$, 1.74 1.79 $(\mathrm{m}, 2 \mathrm{H}), 1.37 \sim 1.42(\mathrm{~m}, 2 \mathrm{H}), 1.18 \sim 1.27(\mathrm{~m}, 8 \mathrm{H}) .{ }^{13} \mathrm{C}$ NMR (100 MHz, DMSO-d $\left.{ }_{6}\right)$ 
$\delta 144.29,141.89,135.81,131.74,129.12 \times 2,125.18,123.88 \times 2,120.75,118.97,113.39$, $103.11,97.90,75.13,61.32,60.68,49.27,32.44,29.67,28.73,28.40,25.74,25.37$. API-ES $(m / z)$ : $612.3[\mathrm{M}+\mathrm{Na}]^{+}$.

Compound 30: ${ }^{1} \mathrm{H}$ NMR (400 MHz, DMSO-d 6 ) $\delta 7.90(\mathrm{~s}, 1 \mathrm{H}), 7.62 \sim 7.66(\mathrm{~m}, 4 \mathrm{H})$, $5.35(\mathrm{~s}, 2 \mathrm{H}), 4.43(\mathrm{~s}, 2 \mathrm{H}), 4.34(\mathrm{~s}, 1 \mathrm{H}), 4.29(\mathrm{t}, 2 \mathrm{H}, \mathrm{J}=4.8 \mathrm{~Hz}), 3.38(\mathrm{~m}, 2 \mathrm{H}), 1.73 \sim 1.78(\mathrm{~m}, 2 \mathrm{H})$, $1.37 \sim 1.40(\mathrm{~m}, 2 \mathrm{H}), 1.22 \sim 1.24(\mathrm{~m}, 8 \mathrm{H}), 1.16 \sim 1.90(\mathrm{~m}, 2 \mathrm{H}) .{ }^{13} \mathrm{C}$ NMR $\left(100 \mathrm{MHz}, \mathrm{DMSO}-d_{6}\right) \delta$ $144.29,141.87,135.76,131.77,129.13 \times 2,125.18,123.90 \times 2,120.75,118.96,113.42,103.13$, $97.83,75.15,61.29,60.66,49.23,32.45,29.64,29.10,28.83,28.28,25.71,25.40$. API-ES $(m / z)$ : $626.5[\mathrm{M}+\mathrm{Na}]^{+}$.

Compound 31: ${ }^{1} \mathrm{H}$ NMR (400 MHz, DMSO-d $) \delta 7.90(\mathrm{~s}, 1 \mathrm{H}), 7.63 \sim 7.66(\mathrm{~m}, 4 \mathrm{H})$, $5.34(\mathrm{~s}, 2 \mathrm{H}), 4.42(\mathrm{~s}, 2 \mathrm{H}), 4.47(\mathrm{~s}, 1 \mathrm{H}), 4.29(\mathrm{t}, 2 \mathrm{H}, J=4.8 \mathrm{~Hz}), 3.36(\mathrm{t}, 2 \mathrm{H}, J=4.4 \mathrm{~Hz})$, $1.72 \sim 1.77(\mathrm{~m}, 2 \mathrm{H}), 1.36 \sim 1.40(\mathrm{~m}, 2 \mathrm{H}), 1.20 \sim 1.24(\mathrm{~m}, 10 \mathrm{H}), 1.16 \sim 1.19(\mathrm{~m}, 2 \mathrm{H}) .{ }^{13} \mathrm{C} \mathrm{NMR}$ $\left(100 \mathrm{MHz}\right.$, DMSO-d $d_{6} \delta 144.30,141.87,135.74,131.78,129.14 \times 2,125.18,123.92 \times 2,120.75$, $118.96,113.45,103.12,97.81,75.15,61.28,60.65,49.21,32.46,29.62,28.91,28.84,28.75,28.28$, 25.70, 25.41. API-ES $(m / z): 640.2[\mathrm{M}+\mathrm{Na}]^{+}$.

\subsubsection{Synthesis of Compounds 33-41}

Synthesis of the title compound was accomplished according to a previously reported procedure [16]. EDC (0.747 $\mathrm{g}, 3.90 \mathrm{mmol})$ and DMAP $(0.293 \mathrm{~g}, 2.4 \mathrm{mmol})$ were added to a solution of Boc-Glu-OH $(1.00 \mathrm{~g}, 3.90 \mathrm{mmol})$ in $\mathrm{CH}_{2} \mathrm{Cl}_{2}(5 \mathrm{~mL})$, followed by $23-31$ ( $3 \mathrm{mmol})$. The mixture was stirred at $\mathrm{rt}$ for $6 \mathrm{~h}$, followed by extraction with $\mathrm{H}_{2} \mathrm{O}(3 \times 5 \mathrm{~mL})$ and drying with $\mathrm{MgSO}_{4}$. After the drying agent was filtered, the solvent was removed under reduced pressure. The residues were purified by column chromatography to obtain compounds 33-41.

Compound 33: ${ }^{1} \mathrm{H}$ NMR (400 MHz, DMSO-d $): \delta 7.99(\mathrm{~s}, 1 \mathrm{H}), 7.63 \sim 7.65(\mathrm{~m}, 4 \mathrm{H})$, $7.11(\mathrm{~d}, 1 \mathrm{H}, J=5.2 \mathrm{~Hz}), 5.38(\mathrm{~s}, 2 \mathrm{H}), 4.62(\mathrm{t}, 2 \mathrm{H}, J=3.6 \mathrm{~Hz}), 4.45(\mathrm{~s}, 2 \mathrm{H}), 4.40(\mathrm{t}, 2 \mathrm{H}$, $J=3.6 \mathrm{~Hz}), 3.80 \sim 3.84(\mathrm{~m}, 1 \mathrm{H}), 2.29 \sim 2.37(\mathrm{~m}, 2 \mathrm{H}), 1.34 \sim 1.39(\mathrm{~m}, 20 \mathrm{H}) .{ }^{13} \mathrm{C} \mathrm{NMR}(100 \mathrm{MHz}$, DMSO- $\left.d_{6}\right): \delta 171.73,171.18,155.43,144.32,142.11,135.81,131.75,129.14,125.17,124.51$, $120.75,119.41,119.16,118.96,113.45,103.09,97.91,80.29,78.01,75.20,62.26,59.62,53.35$, $48.42,29.76,27.99 \times 2,27.75,27.43 \times 3,25.67$. API-ES $(m / z): 813.3[\mathrm{M}+\mathrm{Na}]^{+}$.

Compound 34: ${ }^{1} \mathrm{H}$ NMR (400 MHz, DMSO-d $): \delta 7.95(\mathrm{~s}, 1 \mathrm{H}), 7.63 \sim 7.66(\mathrm{~m}, 4 \mathrm{H})$, $7.13(\mathrm{~d}, 1 \mathrm{H}, \mathrm{J}=5.2 \mathrm{~Hz}), 5.37(\mathrm{~s}, 2 \mathrm{H}), 4.44(\mathrm{~s}, 2 \mathrm{H}), 4.42(\mathrm{t}, 2 \mathrm{H}, \mathrm{J}=4.4 \mathrm{~Hz}), 4.00 \sim 4.04(\mathrm{~m}, 2 \mathrm{H})$, $3.84 \sim 3.88(\mathrm{~m}, 1 \mathrm{H}), 2.35 \sim 2.38(\mathrm{~m}, 2 \mathrm{H}), 2.10 \sim 2.14(\mathrm{~m}, 2 \mathrm{H}), 1.34 \sim 1.39(\mathrm{~m}, 20 \mathrm{H}) .{ }^{13} \mathrm{C} \mathrm{NMR}$ $\left(100 \mathrm{MHz}, \mathrm{DMSO}-d_{6}\right): \delta 172.00,171.25,155.46,144.30,142.00,135.78,131.76,129.12,125.17$, $124.11,120.74,119.38,119.13,118.95,113.34,103.06,97.88,80.30,78.00,75.17,61.27,60.82$, 53.37, 46.33, 29.84, 28.85, $27.99 \times 2,27.75,27.46 \times 3,25.79$. API-ES $(m / z): 827.5[\mathrm{M}+\mathrm{Na}]^{+}$.

Compound 35: ${ }^{1} \mathrm{H}$ NMR (400 MHz, DMSO-d $\left.d_{6}\right): \delta 7.92(\mathrm{~s}, 1 \mathrm{H}), 7.62 \sim 7.65(\mathrm{~m}, 4 \mathrm{H})$, $7.13(\mathrm{~d}, 1 \mathrm{H}, J=5.2 \mathrm{~Hz}), 5.36(\mathrm{~s}, 2 \mathrm{H}), 4.44(\mathrm{~s}, 2 \mathrm{H}), 4.35(\mathrm{t}, 2 \mathrm{H}, J=4.4 \mathrm{~Hz}), 4.01 \sim 4.04(\mathrm{~m}, 2 \mathrm{H})$, $3.83 \sim 3.87(\mathrm{~m}, 1 \mathrm{H}), 2.35 \sim 2.40(\mathrm{~m}, 2 \mathrm{H}), 1.75 \sim 1.87(\mathrm{~m}, 4 \mathrm{H}), 1.50 \sim 1.54(\mathrm{~m}, 2 \mathrm{H}), 1.34 \sim 1.41(\mathrm{~m}, 20 \mathrm{H})$. ${ }^{13} \mathrm{C}$ NMR $\left(100 \mathrm{MHz}, \mathrm{DMSO}-d_{6}\right): \delta 172.07,171.27,155.44,144.29,141.93,135.76,131.76$, $129.12,125.17,123.94,120.74,119.37,119.12,118.95,113.35,103.07,97.86,80.26,77.98,75.16$, $63.08,61.29,53.36,48.81,29.87,28.00,27.76,27.45 \times 3,26.30,25.82,25.03$. API-ES $(m / z)$ : $841.7[\mathrm{M}+\mathrm{Na}]^{+}$.

Compound 36: ${ }^{1} \mathrm{H}$ NMR (400 MHz, DMSO- $\left.d_{6}\right): \delta 7.91(\mathrm{~s}, 1 \mathrm{H}), 7.62 \sim 7.64(\mathrm{~m}, 4 \mathrm{H})$, $7.12(\mathrm{~d}, 1 \mathrm{H}, J=5.2 \mathrm{~Hz}), 5.37(\mathrm{~s}, 2 \mathrm{H}), 4.45(\mathrm{~s}, 2 \mathrm{H}), 4.32(\mathrm{t}, 2 \mathrm{H}, J=4.4 \mathrm{~Hz}), 3.98 \sim 4.04(\mathrm{~m}, 2 \mathrm{H})$, 2.34 2.39 (m, 2H), 1.79 1.82 (m, 3H), 1.58 1.60 (m, 2H), 1.35 1.39 (m, 20H), 1.24 1.29 (m, 2H). ${ }^{13} \mathrm{C}$ NMR $\left(100 \mathrm{MHz}, \mathrm{DMSO}-d_{6}\right): \delta 172.05,171.27,155.44,144.27,141.89,135.77,131.74$, $129.11,125.17,123.86,120.74,119.40,119.14,118.95,113.32,103.06,97.88,80.25,77.97,75.13$, $63.45,61.29,53.37,49.04,30.59,29.88,29.22,27.99 \times 2,27.45,27.44,27.37,27.31,27.31,25.85$, 22.19. API-ES $(m / z): 855.7[\mathrm{M}+\mathrm{Na}]^{+}$.

Compound 37: ${ }^{1} \mathrm{H}$ NMR (400 MHz, DMSO-d $): \delta 7.90(\mathrm{~s}, 1 \mathrm{H}), 7.63 \sim 7.65(\mathrm{~m}, 4 \mathrm{H})$, $7.12(\mathrm{~d}, 1 \mathrm{H}, J=5.2 \mathrm{~Hz}), 5.36(\mathrm{~s}, 2 \mathrm{H}), 4.44(\mathrm{~s}, 2 \mathrm{H}), 4.30(\mathrm{t}, 2 \mathrm{H}, J=4.8 \mathrm{~Hz}), 3.98 \sim 4.00(\mathrm{~m}, 2 \mathrm{H})$, $2.34 \sim 2.38(\mathrm{~m}, 2 \mathrm{H}), 1.74 \sim 1.80(\mathrm{~m}, 3 \mathrm{H}), 1.52 \sim 1.56(\mathrm{~m}, 2 \mathrm{H}), 1.40 \sim 1.43(\mathrm{~m}, 6 \mathrm{H}), 1.37 \sim 1.38(\mathrm{~m}, 14 \mathrm{H})$, $1.33 \sim 1.34(\mathrm{~m}, 2 \mathrm{H}), 1.20 \sim 1.25(\mathrm{~m}, 2 \mathrm{H}) .{ }^{13} \mathrm{C}$ NMR $\left(100 \mathrm{MHz}, \mathrm{DMSO}-d_{6}\right): \delta 172.06,171.26$, 
$155.42,144.26,141.85,135.74,131.74,129.10,125.16,123.85,120.72,119.36,119.11,118.94$, $113.33,103.06,97.84,80.23,77.94,75.13,63.59,61.29,53.34,49.11,30.59,29.89,29.50,27.99$, $27.82,27.75,27.45,27.35,27.32,25.84,25.33,24.65$. API-ES $(m / z): 869.7[\mathrm{M}+\mathrm{Na}]^{+}$.

Compound 38: ${ }^{1} \mathrm{H}$ NMR $\left(400 \mathrm{MHz}, \mathrm{DMSO}-d_{6}\right): \delta 7.90(\mathrm{~s}, 1 \mathrm{H}), 7.63 \sim 7.66(\mathrm{~m}, 4 \mathrm{H})$, $7.12(\mathrm{~d}, 1 \mathrm{H}, J=5.2 \mathrm{~Hz}), 5.36(\mathrm{~s}, 2 \mathrm{H}), 4.44(\mathrm{~s}, 2 \mathrm{H}), 4.30(\mathrm{t}, 2 \mathrm{H}, J=4.4 \mathrm{~Hz}), 3.98 \sim 4.00(\mathrm{~m}, 2 \mathrm{H})$, 2.33 2.38 (m, 2H), 1.74 1.80 (m, 3H), 1.53 1.55 (m, 2H), 1.38 1.43 (m, 20H), 1.27 1.29 (m, $4 \mathrm{H})$, $1.20 \sim 1.22(\mathrm{~m}, 2 \mathrm{H}) .{ }^{13} \mathrm{C}$ NMR $\left(100 \mathrm{MHz}\right.$, DMSO- $\left.d_{6}\right): \delta 172.06,171.26,155.41,144.25,141.85$, $135.75,131.73,129.10,125.16,123.83,120.72,119.38,119.13,118.93,113.31,103.04,97.86$, $80.21,77.94,75.12,63.68,61.29,53.34,49.17,30.59,29.89,29.55,27.99,27.94,27.91,27.74$, 27.44, 27.34, 27.31, 25.86, 25.60, 25.10. API-ES $(m / z): 883.5\left[\mathrm{M}+\mathrm{Na}^{+}\right.$.

Compound 39: ${ }^{1} \mathrm{H}$ NMR $\left(400 \mathrm{MHz}, \mathrm{DMSO}-d_{6}\right): \delta 7.90(\mathrm{~s}, 1 \mathrm{H}), 7.63 \sim 7.66(\mathrm{~m}, 4 \mathrm{H})$, $7.12(\mathrm{~d}, 1 \mathrm{H}, J=5.2 \mathrm{~Hz}), 5.36(\mathrm{~s}, 2 \mathrm{H}), 4.44(\mathrm{~s}, 2 \mathrm{H}), 4.30(\mathrm{t}, 2 \mathrm{H}, J=4.4 \mathrm{~Hz}), 3.98 \sim 4.00(\mathrm{~m}, 2 \mathrm{H})$, 2.33 2.38 (m, 2H), 1.75 1.78 (m, 3H), 1.53 1.55 (m, 2H), 1.41 1.43 (m, 20H), 1.26 1.28 (m, 6H), 1.18 1.21 (m, 2H). ${ }^{13} \mathrm{C}$ NMR (100 MHz, DMSO- $\left.d_{6}\right): \delta 172.04,171.25,155.40,144.25,141.83$, $135.73,131.73,129.09,125.16,123.84,120.72,119.36,119.10,118.93,113.31,103.03,97.84$, $80.21,77.92,75.12,63.68,61.28,58.96,53.33,49.17,30.59,29.88,29.59,28.36,28.16,27.99$, 27.74, 27.44, 27.35, 27.32, 25.85, 25.62, 25.15. API-ES $(m / z): 897.5[\mathrm{M}+\mathrm{Na}]^{+}$.

Compound 40: ${ }^{1} \mathrm{H}$ NMR $\left(400 \mathrm{MHz}, \mathrm{DMSO}-d_{6}\right): \delta 7.90(\mathrm{~s}, 1 \mathrm{H}), 7.62 \sim 7.64(\mathrm{~m}, 4 \mathrm{H})$, $7.11(\mathrm{~d}, 1 \mathrm{H}, J=5.2 \mathrm{~Hz}), 5.35(\mathrm{~s}, 2 \mathrm{H}), 4.43(\mathrm{~s}, 2 \mathrm{H}), 4.29(\mathrm{t}, 2 \mathrm{H}, J=4.4 \mathrm{~Hz}), 3.98 \sim 4.00(\mathrm{~m}, 2 \mathrm{H})$, 2.32 2.38 (m, 2H), 1.74 1.78 (m, 3H), 1.52 1.55 (m, 2H), 1.41 1.43 (m, 12H), 1.37 1.38 (m, 14H), 1.25 1.27 $(\mathrm{m}, 4 \mathrm{H}) .{ }^{13} \mathrm{C}$ NMR $\left(100 \mathrm{MHz}\right.$, DMSO- $\left.d_{6}\right): \delta 172.05,171.26,155.40,144.26,141.84$, $135.72,131.74,129.10,125.16,123.85,120.72,119.33,119.08,118.93,113.34,103.05,97.82$, $80.22,77.93,75.13,63.72,61.27,58.96,53.31,49.18,30.59,29.89,29.59,28.63,28.45,28.20$, 27.99, 27.76, 27.46, 27.36, 27.33, 25.84, 25.66, 25.21. API-ES $(m / z): 911.5[\mathrm{M}+\mathrm{Na}]^{+}$.

Compound 41: ${ }^{1} \mathrm{H}$ NMR $\left(400 \mathrm{MHz}\right.$, DMSO- $\left.d_{6}\right): \delta 8.52(\mathrm{~m}, 2 \mathrm{H}), 7.90(\mathrm{~s}, 1 \mathrm{H}), 7.63 \sim 7.64(\mathrm{~m}, 4 \mathrm{H})$, $5.35(\mathrm{~s}, 2 \mathrm{H}), 4.43(\mathrm{~s}, 2 \mathrm{H}), 4.29(\mathrm{t}, 2 \mathrm{H}, J=4.4 \mathrm{~Hz}), 3.97 \sim 4.00(\mathrm{~m}, 2 \mathrm{H}), 2.32 \sim 2.38(\mathrm{~m}, 2 \mathrm{H})$, $1.73 \sim 1.78(\mathrm{~m}, 3 \mathrm{H}), 1.52 \sim 1.55(\mathrm{~m}, 2 \mathrm{H}), 1.41 \sim 1.43(\mathrm{~m}, 10 \mathrm{H}), 1.37 \sim 1.38(\mathrm{~m}, 16 \mathrm{H}), 1.24 \sim 1.26(\mathrm{~m}, 6 \mathrm{H})$ ${ }^{13} \mathrm{C}$ NMR $\left(100 \mathrm{MHz}, \mathrm{DMSO}-d_{6}\right): \delta 172.05,171.26,155.40,144.26,141.84,135.72,131.74$, 129.10, 125.16, 123.85, 120.72, 119.33, 119.08, 118.93, 113.35, 103.05, 97.82, 80.23, 77.94, 75.13, $63.73,61.27,58.96,53.32,49.18,30.60,29.89,29.61,28.72,28.68,28.52,28.26,28.01,27.76$, 27.46, 27.37, 27.34, 25.84, 25.67, 25.24. API-ES $(m / z): 925.7[\mathrm{M}+\mathrm{Na}]^{+}$.

\subsubsection{Synthesis of Conjugates $\mathbf{4 2 - 5 0}$}

Synthesis of the title compound was accomplished according to a previously reported procedure [16]. Compounds 33-41 were added to a mixture solution of $\mathrm{CH}_{2} \mathrm{Cl}_{2}(3 \mathrm{~mL})$ and TFA ( $3 \mathrm{~mL}$ ). The mixture was stirred at $\mathrm{rt}$ for $12 \mathrm{~h}$, after which the solvent was removed in vacuo. The residues were purified by column chromatography to obtain conjugates 42-50.

Conjugate 42: ${ }^{1} \mathrm{H}$ NMR (400 MHz, DMSO- $\left.d_{6}\right): \delta 8.50(\mathrm{~d}, 2 \mathrm{H}, J=4.8 \mathrm{~Hz}), 7.96(\mathrm{~s}, 1 \mathrm{H})$, 7.61 7.63 (m, 4H), $5.36(\mathrm{~s}, 2 \mathrm{H}), 4.36 \sim 4.41(\mathrm{~m}, 4 \mathrm{H}), 3.98 \sim 4.01(\mathrm{~m}, 2 \mathrm{H}), 3.75(\mathrm{~s}, 1 \mathrm{H}), 2.42 \sim 2.50(\mathrm{~m}, 2 \mathrm{H})$, 1.95 2.10 (m, 2H). ${ }^{13} \mathrm{C}$ NMR (100 MHz, DMSO- $\left.d_{6}\right): \delta 172.32,170.62,144.59,142.35,141.98$, $136.02,131.95,129.36,125.29,124.88,120.92,119.62,119.37,119.13,113.69,103.32,98.03$, 75.35, 62.45, 61.35, 52.28, 29.80, 28.25. API-ES $(m / z): 656.2[\mathrm{M}+\mathrm{Na}]^{+}$.

Conjugate 43: ${ }^{1} \mathrm{H}$ NMR $\left(400 \mathrm{MHz}, \mathrm{DMSO}-d_{6}\right): \delta 8.54(\mathrm{~m}, 2 \mathrm{H}), 7.96(\mathrm{~s}, 1 \mathrm{H}), 7.64(\mathrm{~m}, 4 \mathrm{H})$, $5.35(\mathrm{~s}, 2 \mathrm{H}), 4.39 \sim 4.41(\mathrm{~m}, 4 \mathrm{H}), 3.98(\mathrm{t}, 2 \mathrm{H}, J=3.6 \mathrm{~Hz}), 3.59(\mathrm{~s}, 1 \mathrm{H}), 2.45 \sim 2.55(\mathrm{~m}, 2 \mathrm{H})$, $2.09(\mathrm{t}, 2 \mathrm{H}, J=3.6 \mathrm{~Hz}), 1.91 \sim 2.02(\mathrm{~m}, 2 \mathrm{H}) .{ }^{13} \mathrm{C}$ NMR $\left(100 \mathrm{MHz}, \mathrm{DMSO}-d_{6}\right): \delta 172.13,170.98$, $144.45,142.13,141.98,135.86,131.89,129.26,125.25,124.32,120.84,119.44,119.19,119.05$, 113.57, 103.24, 97.90, 75.30, 61.33, 60.97, 46.36, 29.56, 28.94, 25.73. API-ES $(m / z)$ : $670.2[\mathrm{M}+\mathrm{Na}]^{+}$.

Conjugate 44: ${ }^{1} \mathrm{H}$ NMR $\left(400 \mathrm{MHz}\right.$, DMSO- $\left.d_{6}\right): \delta 8.53(\mathrm{~m}, 2 \mathrm{H}), 7.92(\mathrm{~s}, 1 \mathrm{H}), 7.63 \sim 7.65(\mathrm{~m}, 4 \mathrm{H})$, $5.35(\mathrm{~s}, 2 \mathrm{H}), 4.35 \sim 4.40(\mathrm{~m}, 4 \mathrm{H}), 4.02 \sim 4.04(\mathrm{~m}, 2 \mathrm{H}), 3.61(\mathrm{~s}, 1 \mathrm{H}), 2.40 \sim 2.45(\mathrm{~m}, 2 \mathrm{H}), 1.97 \sim 2.01(\mathrm{~m}, 2 \mathrm{H})$, $1.51(\mathrm{~m}, 2 \mathrm{H}), 1.40 \sim 1.43(\mathrm{~m}, 2 \mathrm{H}) .{ }^{13} \mathrm{C}$ NMR $\left(100 \mathrm{MHz}, \mathrm{DMSO}-d_{6}\right): \delta 171.94,168.33,144.36$, $142.08,141.96,135.74,131.84,129.59,125.21,124.07,120.76,119.30,119.05,118.98,113.49$, 103.17, 97.80, 75.26, 63.29, 61.27, 51.59, 28.95, 27.41, 26.30, 25.02. API-ES $(m / z)$ : $684.3[\mathrm{M}+\mathrm{Na}]^{+}$. 
Conjugate 45: ${ }^{1} \mathrm{H}$ NMR $\left(400 \mathrm{MHz}\right.$, DMSO- $\left.d_{6}\right): \delta 8.51(\mathrm{~m}, 2 \mathrm{H}), 7.90(\mathrm{~s}, 1 \mathrm{H}), 7.62 \sim 7.66(\mathrm{~m}, 4 \mathrm{H})$, $5.34(\mathrm{~s}, 2 \mathrm{H}), 4.39 \sim 4.41(\mathrm{~m}, 2 \mathrm{H}), 4.30(\mathrm{t}, 2 \mathrm{H}, J=4.4 \mathrm{~Hz}), 3.99(\mathrm{t}, 2 \mathrm{H}, J=4.4 \mathrm{~Hz}), 3.68(\mathrm{~s}, 1 \mathrm{H})$, 2.39 2.45 (m, 2H), 1.91 2.03 (m, 2H), 1.75 1.80 (m, 2H), 1.56 1.61 (m, 2H), 1.24 1.27 (m, 2H). ${ }^{13} \mathrm{C}$ NMR $\left(100 \mathrm{MHz}, \mathrm{DMSO}-d_{6}\right): \delta 171.96,170.35,144.38,142.07,141.96,135.77,131.86$, 129.20, 125.23, 124.01, 120.79, 119.34, 119.08, 119.00, 113.53, 103.19, 97.81, 75.24, 63.76, 61.31, 51.91, 29.41, 29.27, 27.39, 25.53, 22.24. API-ES $(m / z): 698.0[\mathrm{M}+\mathrm{Na}]^{+}$.

Conjugate 46: ${ }^{1} \mathrm{H}$ NMR $\left(400 \mathrm{MHz}\right.$, DMSO- $\left.d_{6}\right): \delta 8.50(\mathrm{~m}, 2 \mathrm{H}), 7.90(\mathrm{~s}, 1 \mathrm{H}), 7.63 \sim 7.67(\mathrm{~m}, 4 \mathrm{H})$, $5.34(\mathrm{~s}, 2 \mathrm{H}), 4.41(\mathrm{~m}, 2 \mathrm{H}), 4.29(\mathrm{t}, 2 \mathrm{H}, J=4.8 \mathrm{~Hz}), 3.99(\mathrm{t}, 2 \mathrm{H}, J=4.4 \mathrm{~Hz}), 3.69(\mathrm{~s}, 1 \mathrm{H})$, 2.40 2.55 (m, 2H), 1.91 2.04 (m, 2H), 1.75 1.78 (m, 2H), 1.51 1.56 (m, 2H), 1.29 1.32 (m, 2H), 1.19 1.23 (m, 2H). ${ }^{13} \mathrm{C}$ NMR (100 MHz, DMSO- $\left.d_{6}\right): \delta 171.92,170.39,144.33,142.02$, $141.89,135.73,131.82,129.16,125.20,123.96,120.76,119.29,119.04,118.97,113.48,103.16$, $97.78,75.21,63.84,61.28,51.89,29.50,29.39,27.81,25.53,25.35,24.66$. API-ES $(m / z)$ : $712.5[\mathrm{M}+\mathrm{Na}]^{+}$.

Conjugate 47: ${ }^{1} \mathrm{H}$ NMR $\left(400 \mathrm{MHz}\right.$, DMSO- $\left.d_{6}\right): \delta 8.51(\mathrm{~m}, 2 \mathrm{H}), 7.90(\mathrm{~s}, 1 \mathrm{H}), 7.63(\mathrm{~m}, 4 \mathrm{H})$, $5.34(\mathrm{~s}, 2 \mathrm{H}), 4.41(\mathrm{~m}, 2 \mathrm{H}), 4.28(\mathrm{t}, 2 \mathrm{H}, J=4.0 \mathrm{~Hz}), 3.99(\mathrm{t}, 2 \mathrm{H}, J=4.4 \mathrm{~Hz}), 3.74(\mathrm{~s}, 1 \mathrm{H})$, 2.44 2.52 (m, 2H), 1.94 2.02 (m, 2H), $1.73(\mathrm{~m}, 2 \mathrm{H}), 1.53(\mathrm{~m}, 2 \mathrm{H}), 1.27(\mathrm{~m}, 4 \mathrm{H}), 1.18(\mathrm{~m}, 2 \mathrm{H})$. ${ }^{13} \mathrm{C}$ NMR (100 MHz, DMSO- $\left.d_{6}\right): \delta 172.00,170.68,144.41,142.07,141.96,135.82,131.88$, $129.23,125.26,124.06,120.83,119.41,119.16,119.04,113.57,103.23,97.86,75.26,64.03,61.34$, 51.90, 29.62, 29.41, 28.17, 27.97, 25.67, 25.54, 25.17. API-ES $(m / z): 726.3[\mathrm{M}+\mathrm{Na}]^{+}$.

Conjugate 48: ${ }^{1} \mathrm{H}$ NMR $\left(400 \mathrm{MHz}, \mathrm{DMSO}-d_{6}\right): \delta 8.50(\mathrm{~m}, 2 \mathrm{H}), 7.90(\mathrm{~s}, 1 \mathrm{H}), 7.64(\mathrm{~m}, 4 \mathrm{H})$, $5.34(\mathrm{~s}, 2 \mathrm{H}), 4.41(\mathrm{~m}, 2 \mathrm{H}), 4.28(\mathrm{t}, 2 \mathrm{H}, J=3.6 \mathrm{~Hz}), 3.98(\mathrm{t}, 2 \mathrm{H}, J=4.4 \mathrm{~Hz}), 3.71(\mathrm{~s}, 1 \mathrm{H})$, 2.43 2.50 (m, 2H), 1.93 2.02 (m, 2H), $1.74(\mathrm{~m}, 2 \mathrm{H}), 1.53(\mathrm{~m}, 2 \mathrm{H}), 1.24(\mathrm{~m}, 6 \mathrm{H}), 1.13 \sim 1.16(\mathrm{~m}, 2 \mathrm{H})$. ${ }^{13} \mathrm{C}$ NMR (100 MHz, DMSO- $\left.d_{6}\right): \delta 172.26,170.33,144.36,142.02,141.91,135.78,131.84$, $129.19,125.22,124.01,120.79,119.36,119.11,118.90,113.51,103.17,97.83,75.22,63.88,61.31$, $52.70,29.70,29.63,28.43,28.22,28.03,25.91,25.68,25.22$. API-ES $(\mathrm{m} / \mathrm{z}): 740.3[\mathrm{M}+\mathrm{Na}]^{+}$.

Conjugate 49: ${ }^{1} \mathrm{H}$ NMR $\left(400 \mathrm{MHz}, \mathrm{DMSO}-d_{6}\right): \delta 8.52(\mathrm{~m}, 2 \mathrm{H}), 7.90(\mathrm{~s}, 1 \mathrm{H}), 7.60 \sim 7.64(\mathrm{~m}, 4 \mathrm{H})$, $5.35(\mathrm{~s}, 2 \mathrm{H}), 4.46(\mathrm{~m}, 2 \mathrm{H}), 4.29(\mathrm{t}, 2 \mathrm{H}, J=4.4 \mathrm{~Hz}), 3.97 \sim 3.99(\mathrm{~m}, 3 \mathrm{H}), 2.35 \sim 2.41(\mathrm{~m}, 2 \mathrm{H})$, $1.87 \sim 1.96(\mathrm{~m}, 2 \mathrm{H}), 1.74 \sim 1.76(\mathrm{~m}, 2 \mathrm{H}), 1.50 \sim 1.56(\mathrm{~m}, 4 \mathrm{H}), 1.40 \sim 1.42(\mathrm{~m}, 6 \mathrm{H}), 1.18 \sim 1.19(\mathrm{~m}, 2 \mathrm{H})$. ${ }^{13} \mathrm{C}$ NMR (100 MHz, DMSO- $\left.d_{6}\right): \delta 172.49,171.09,144.28,142.02,141.90,135.89,131.69$, 129.13, 125.17, 123.86, 120.77, 119.58, 119.33, 118.98, 113.30, 103.04, 97.99, 75.10, 63.75, $61.35,50.67,29.93,29.71,28.75,28.56,28.32,28.10,27.49,25.78,25.35$. API-ES $(m / z)$ : $754.3[\mathrm{M}+\mathrm{Na}]^{+}$.

Conjugate 50: ${ }^{1} \mathrm{H}$ NMR $\left(400 \mathrm{MHz}, \mathrm{DMSO}-d_{6}\right): \delta 8.54(\mathrm{~m}, 2 \mathrm{H}), 7.91(\mathrm{~s}, 1 \mathrm{H}), 7.62(\mathrm{~m}, 4 \mathrm{H})$, $5.35(\mathrm{~s}, 2 \mathrm{H}), 4.43(\mathrm{~m}, 2 \mathrm{H}), 4.28(\mathrm{t}, 2 \mathrm{H}, J=4.4 \mathrm{~Hz}), 4.00(\mathrm{t}, 2 \mathrm{H}, J=4.4 \mathrm{~Hz}), 3.94(\mathrm{~s}, 1 \mathrm{H})$, 2.53 2.57 (m, 1H), 2.39 2.44 (m, 1H), 2.02 2.07 (m, 2H), 1.72 1.77 (m, 2H), 1.53 1.55 (m, 2H), $1.43(\mathrm{~m}, 8 \mathrm{H}), 1.21 \sim 1.24(\mathrm{~m}, 2 \mathrm{H}) .{ }^{13} \mathrm{C}$ NMR $\left(100 \mathrm{MHz}, \mathrm{DMSO}-d_{6}\right): \delta 172.10,168.19,144.36$, $141.98,141.15,135.87,131.81,129.19,125.24,124.02,120.82,119.53,119.27,119.03,113.45$, $103.12,97.95,75.19,64.14,61.36,51.68,29.71,29.04,28.83,28.78,28.62,28.36,28.08,27.38$, 25.78, 25.35. API-ES $(m / z): 768.3[\mathrm{M}+\mathrm{Na}]^{+}$.

\subsection{Insecticidal Activity of Nine Conjugates against P. xylostella}

Bioassay of insecticidal activity of nine conjugates against $P$. xylostellaas was described previously $[15,16]$. The insecticidal activity of compounds $\mathbf{4 2 - 5 0}$ and chlorfenapyragainst $P$. xylostella larvae was determined by mixing them with diet. Nine conjugates were dissolved in methanol and acetone $(10 / 90, v / v)$ at three concentration levels $(0.5,1$, and $2 \mathrm{mM})$. Each test solution $(1 \mathrm{~mL})$ and artificial diet $(1 \mathrm{~g})$ were placed in Petri dishes $(6 \mathrm{~cm}$ in diameter). An artificial diet $(1 \mathrm{~g})$ containing methanol and acetone $(1 \mathrm{~mL}, 10 / 90, v / v)$ was used as control. Ten P. xylostella larvae were introduced into each dish. P. xylostella was cultured at $26^{\circ} \mathrm{C}, 70 \%$ relative humidity, and $16: 8 \mathrm{~h}$ photoperiod (light/dark). The experiments were repeated three times. The mortality of $P$. xylostella was measured $24 \mathrm{~h}$ after culture.

\subsection{Sap Collection from Ricinus communis L. Seedlings and Analysis}

Castor bean seeds (R. communis L.) were obtained from the Agricultural Science Academy of Zibo, Shandong, China, and grown as previously described [19]. Six days 
after sowing, average-sized seedlings were selected for the experiments. Phloem sap was collected from the upper part of the hypocotyl according to previously described methods $[19,20]$. The endosperm of seedlings was carefully removed without bending or crushing the cotyledons. These latter organs were incubated in a buffer solution containing $20 \mathrm{mM}$ MES (pH 5.0), $0.25 \mathrm{mM} \mathrm{MgCl}$, and $0.5 \mathrm{mM} \mathrm{CaCl}_{2}$ supplemented with $200 \mu \mathrm{M}$ 42-45 or $200 \mu \mathrm{M}$ chlorfenapyr. After $1 \mathrm{~h}$ of incubation, the hypocotyl was severed in the hook region for phloem exudation, and the collected sap was stored in ice until analysis.

The phloem sap was analyzed by an HPLC (HP 1260 system, Agilent Technologies, Santa Clara, CA, USA, equipped with a quaternary pump, autosampler, and UV-visible detector)after dilution with methanol (phloem sap/methanol, $1 / 2$ or $1 / 3, v / v)$. Separations were performed with a C18 reversed-phase column $(5 \mu \mathrm{m}, 250 \times 4.6 \mathrm{~mm}$ i. $\mathrm{d}$, Agilent Co., Santa Clara, CA, USA) at $25^{\circ} \mathrm{C}$. The mobile solvent system consisted of acetonitrile and water containing $0.1 \%$ trifluoroacetic acid $(80 / 20, v / v$ for $\mathbf{4 2 - 4 5}$ and chlorfenapyr) at a flow rate of $0.5 \mathrm{~mL} / \mathrm{min}$. The detector wavelength was $205 \mathrm{~nm}$ for $\mathbf{4 2 - 4 5}$ and chlorfenapyr. External calibration was used to determine their concentrations. A series of standard solutions $(0.5,1,5,10,25$, and $50 \mu \mathrm{M})$ for linearities was prepared in methanol. Their linear equations were as follows: $y=7.4216 x+2.2817\left(R^{2}=0.9996\right)$ for $42, y=12.959 x+3.7681$ $\left(R^{2}=0.9998\right)$ for $43, y=3.0498 x+1.5868\left(R^{2}=0.9922\right)$ for $44, y=5.1043 x+5.6425\left(R^{2}=0.9917\right)$ for 45 , and $y=30.993 x+25.683\left(R^{2}=0.9989\right)$ for chlorfenapyr.

\subsection{Metabolism of Conjugates 42 and 43 in P. xylostella}

The metabolism of conjugates 42 and 43 in P. xylostella as was previously described [21-23]. The in vivo mechanism of compounds 42 and 43 in P. xylostella larvae was determined by mixing them with diet. The preparation of the artificial diet with $0.5 \mathrm{mM}$ of tested conjugates was mentioned above. The experiments were repeated three times. After $24 \mathrm{~h}$, the $P$. xylostella were removed, washed with normal saline, added to $0.1 \mathrm{~mL}$ methanol, and homogenized in an ice bath. After ultrasonic extraction for $10 \mathrm{~min}$, the worms were centrifuged at $8000 \mathrm{r}$ and $4{ }^{\circ} \mathrm{C}$ for $10 \mathrm{~min}$. The supernatant was taken and stored at $-20{ }^{\circ} \mathrm{C}$ for measurement.

The supernatant was analyzed by LC-MS/MS. Chromatographic conditions: metal straight pipe, flow rate $0.3 \mathrm{~mL} / \mathrm{min}$; column temperature $40{ }^{\circ} \mathrm{C}$; injection volume $3 \mu \mathrm{L}$; the mobile phase was B methanol 100\%. Mass spectrum conditions: Pola: ESI-; acquisition time: $1 \mathrm{~min}$; acquisition model: MS resolution: 45,000 (LE); acquisition rang: 50 1500 Da; collision energy $6 \mathrm{eV}$; Lockspary: $0.6 \mathrm{ng} / \mathrm{mL}, 10 \mathrm{uL} / \mathrm{min}, \mathrm{ESI}-\mathrm{m} / z$ : 554.2615, 236.1035. A series of standard solutions $(0.125,0.0625,0.03125,0.015625,0.0078125$ and $0.00390625 \mathrm{mg} / \mathrm{L})$ for linearities was prepared in methanol. Tralopyril linear equation was $\mathrm{y}=57.7547 \times\left(\mathrm{R}^{2}=0.9844\right)($ Over Zero Point $)$.

\subsection{Physicochemical Properties}

The physicochemical properties (molecular mass (MW), the octanol: water partition coefficient $(\log \mathrm{P})$, acid dissociation constant polar $(\mathrm{pKa})$, number of hydrogen bond donors (HBDs), and number of hydrogen bond acceptors (HBAs)) of the conjugates 42-45, tralopyril and chlorfenapyr were predicted using ACD/Percepta 14.0.0 software.

\section{Results and Discussion}

\subsection{Synthesis and Characterization}

The main conjugating method employed between glutamic acid moieties and tralopyril was click chemistry.Terminal alkyne was introduced into tralopyril to afford 4, which reacted with azide intermediates 14-22 (compounds 14-22 were selected and prepared according to the literature) to obtain intermediates 23-31 via click reaction (shown in Schemes 1-3), and the yields reached 80\%. Scheme 4 shows that intermediates 23-31 were coupled with purchased Boc-Glu-OH to afford compounds 33-41 under the condition that EDC and DMAP were used together. Scheme 5 shows that the protecting groups on 
glutamic acidtralopyril conjugates 33-41 were taken off in the presence of TFA in $\mathrm{CH}_{2} \mathrm{Cl}_{2}$ to produce final conjugates $\mathbf{4 2 - 5 0}$ as white solids or liquid in high yields.<smiles>C#CCOCn1c(-c2ccc(Cl)cc2)c(C#N)c(Br)c1C(F)(F)F</smiles>

Scheme 1. Synthetic routes of compound 4. Reagents and conditions: THF, Bromochloromethane (5 equiv), NaH, Propargyl alcohol, $65^{\circ} \mathrm{C}, 48 \mathrm{~h}$.

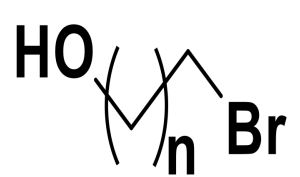

5-13

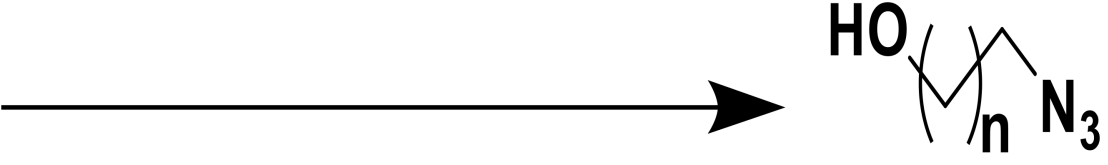

$n=2-10$

Scheme 2. Synthetic routes of compounds 14-22. Reagents and conditions: $\mathrm{NaN}_{3}, \mathrm{n}-\mathrm{Bu}_{4} \mathrm{NBr}, 110{ }^{\circ} \mathrm{C}, 15 \mathrm{~h}$.<smiles>C#CCOCn1c(-c2ccc(Cl)cc2)c(C#N)c(Br)c1C(F)(F)F</smiles>

4<smiles>CCCC(C)C(C)C(C)O</smiles>

14-22<smiles>CCCCn1cc(COCn2c(-c3ccc(Cl)cc3)c(C#N)c(Br)c2C(F)(F)F)nn1</smiles>

23-31

$$
\mathrm{n}=\mathbf{2 - 1 0}
$$

Scheme 3. Synthetic routes of compounds 23-31. Reagents and conditions: $\mathrm{CuSO}_{4} \cdot 5 \mathrm{H}_{2} \mathrm{O}(0.4$ equiv $)$, sodium ascorbate (0.8 equiv), DMF, $60{ }^{\circ} \mathrm{C}, 3 \mathrm{~h}$.<smiles>CCCCn1cc(COCn2c(-c3ccc(Cl)cc3)c(C#N)c(Br)c2C(F)(F)F)nn1</smiles>

23-31

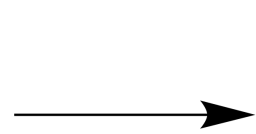

$\mathrm{CF}_{3}$<smiles>CC(=O)O</smiles><smiles>[H][R7]#CC(C)(C)n1cc(COCn2c(-c3ccc(C)cc3)c(C#N)c(Br)c2C(F)(F)F)nn1</smiles><smiles>Cc1ccc(-c2c(C#N)c(Br)c(C(F)(F)F)n2COCc2cn(C(C)(C)OC(=O)C(CCC(=O)OC(C)(C)C)NC(=O)OC(C)(C)C)nn2)cc1</smiles><smiles>CC(C)(C)OC(=O)NC(CCC(=O)O)C(=O)OC(C)(C)C</smiles>

32

33-41

Scheme 4. Synthetic routes of compounds 33-41. Reagents and conditions: $\mathrm{CH}_{2} \mathrm{Cl}_{2}, \mathrm{EDC}, \mathrm{DMAP}$, rt, $6 \mathrm{~h}$. 


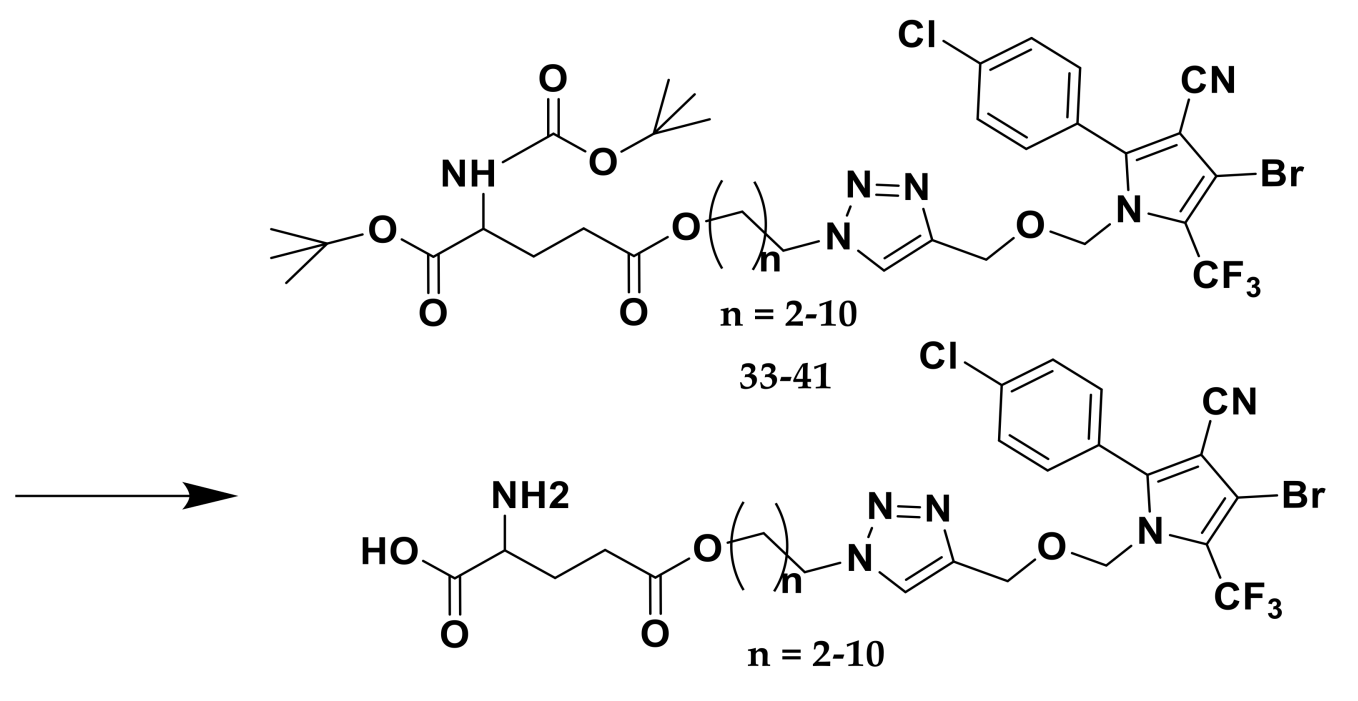

42-50

Scheme 5. Synthetic routes of compounds 42-50. Reagents and conditions: $\mathrm{CH}_{2} \mathrm{Cl}_{2}$, TFA, rt, $12 \mathrm{~h}$.

The structures of compounds 4, 23-31, 33-41, and 42-50 were confirmed by ${ }^{1} \mathrm{H}$, ${ }^{13} \mathrm{C}$ NMR, and API-ES spectra. Signals of terminal alkyne could be observed in the ${ }^{1} \mathrm{H}$ and ${ }^{13} \mathrm{C}$ NMR spectra for compound 4 . The characteristic signals of 1-, 2-, and 3-triazole could be observed in the ${ }^{1} \mathrm{H}$ and ${ }^{13} \mathrm{C}$ NMR spectra of click reaction products 23-31. Compounds 33-41 were further confirmed by the signals of the six methyl groups. The removal of six methyl groups was confirmed by the ${ }^{1} \mathrm{H}$ and ${ }^{13} \mathrm{C}$ NMR spectra of final conjugates 42-50. The mass spectra of the 28 compounds exhibited excellent correlation with the calculated molecular mass.

\subsection{Insecticidal Activity}

The insecticidal activity of conjugates $\mathbf{4 2 - 5 0}$ against $P$. xylostella was preliminarily evaluated, and conjugates $42,43,44$, and 45 exhibited preferable activity under the tested concentrations (Table 1). According to the analysis of the linker structure-activity relationship of the nine conjugates, a longer linker chain showed a lower insecticidal activity. Based on the preliminary activity, the insecticidal activity of conjugates $42,43,44$, and 45 against $P$. xylostella was further evaluated and compared with that of chlorfenapyr over a wide range of concentrations. Table 2 shows that conjugate $\mathbf{4 2}$ showed the highest insecticidal activity, which may be due to the different abilities of the mixed-function oxidases to act on the conjugates of different carbon chain lengths, of which $\mathbf{4 2}$ was the highest. Previous studies showed that specific promoiety introduction that confers phloem mobility to pesticides usually reduced direct biological activity $[2,4,5]$. This phenomenon was also observed in the present work, and the $24 \mathrm{~h} \mathrm{LC}_{50}$ value of these new conjugates was higher than that of tralopyril and chlorfenapyr. Hsu et al. described the strategy of phloem-mobile propesticide to overcome this inherent incompatibility [2]. Conjugates $\mathbf{4 2 - 4 5}$ shared the same O-methylene group on pyrrole $\mathrm{N}$ with chlorfenapyr. Thus, conjugates $\mathbf{4 2}, \mathbf{4 3}, \mathbf{4 4}$, and 45 may have a proinsecticidal mechanism similar to that of chlorfenapyr. This inference needs to be confirmed by obtaining direct evidence in further experiments. 
Table 1. Mortality percentage of $P$. xylostella larvae after $24 \mathrm{~h}$ of exposure to the diet containing the conjugates $\mathbf{4 2 - 5 0 .}$

\begin{tabular}{|c|c|c|c|c|}
\hline & \multicolumn{4}{|c|}{ Concentration } \\
\hline & $2 \mathrm{mM}$ & $1 \mathrm{mM}$ & $0.5 \mathrm{mM}$ & $0.1 \mathrm{mM}$ \\
\hline 42 & $100.00 \pm 0.00 \mathrm{a}$ & $100.0 \pm 0.00 \mathrm{a}$ & $100.00 \pm 0.00 \mathrm{a}$ & $43.33 \pm 0.15 b$ \\
\hline 43 & $100.00 \pm 0.00 \mathrm{a}$ & $100.00 \pm 0.00 \mathrm{a}$ & $76.66 \pm 0.15 b$ & $0.00 \pm 0.00 c$ \\
\hline 44 & $100.00 \pm 0.00 \mathrm{a}$ & $100.00 \pm 0.00 \mathrm{a}$ & $73.33 \pm 0.12 b$ & $0.00 \pm 0.00 c$ \\
\hline 45 & $100.00 \pm 0.00 \mathrm{a}$ & $100.00 \pm 0.00 \mathrm{a}$ & $60.00 \pm 0.26 c$ & $0.00 \pm 0.00 c$ \\
\hline 46 & $100.00 \pm 0.00 \mathrm{a}$ & $83.33 \pm 0.12 b$ & $48.33 \pm 0.19 c$ & $0.00 \pm 0.00 c$ \\
\hline 47 & $100.00 \pm 0.00 \mathrm{a}$ & $23.33 \pm 0.12 c$ & $3.33 \pm 0.06 \mathrm{~d}$ & $0.00 \pm 0.00 \mathrm{c}$ \\
\hline 48 & $100.00 \pm 0.00 \mathrm{a}$ & $3.33 \pm 0.058 \mathrm{~d}$ & $0.00 \pm 0.00 \mathrm{~d}$ & $0.00 \pm 0.00 c$ \\
\hline 49 & $100.00 \pm 0.00 \mathrm{a}$ & $0.00 \pm 0.00 \mathrm{~d}$ & $0.00 \pm 0.00 \mathrm{~d}$ & $0.00 \pm 0.00 c$ \\
\hline 50 & $100.00 \pm 0.00 \mathrm{a}$ & $0.00 \pm 0.00 \mathrm{~d}$ & $0.00 \pm 0.00 \mathrm{~d}$ & $0.00 \pm 0.00 c$ \\
\hline Chlorfenapyr & $100.00 \pm 0.00 \mathrm{a}$ & $100.00 \pm 0.00 \mathrm{a}$ & $100.00 \pm 0.00 \mathrm{a}$ & $100.00 \pm 0.00 a$ \\
\hline
\end{tabular}

Note: English letters of a, b, c and d indicate the difference in $5 \%$ level.

Table 2. The insecticidal activity ofconjugates $42,43,44$, and 45 against $P$. xylostella.

\begin{tabular}{cccc}
\hline & LC $_{\mathbf{5 0}}$ & SE & 95\% Confidence Limits \\
\hline $\mathbf{4 2}$ & $0.2397 \mathrm{mM}$ & 0.0366 & $0.1644 \mathrm{mM}-0.3494 \mathrm{mM}$ \\
$\mathbf{4 3}$ & $0.4413 \mathrm{mM}$ & 0.0647 & $0.3311 \mathrm{mM}-0.5882 \mathrm{mM}$ \\
$\mathbf{4 4}$ & $0.4400 \mathrm{mM}$ & 0.0624 & $0.3309 \mathrm{mM}-0.5849 \mathrm{mM}$ \\
$\mathbf{4 5}$ & $0.4602 \mathrm{mM}$ & 0.0655 & $0.3479 \mathrm{mM}-0.6087 \mathrm{mM}$ \\
Chlorfenapyr & $0.0249 \mathrm{mM}$ & 0.0145 & $0.0079 \mathrm{mM}-0.0784 \mathrm{mM}$ \\
Tralopyril & $0.0224 \mathrm{mM}$ & 0.0601 & $0.0133 \mathrm{mM}-0.0381 \mathrm{mM}$ \\
\hline
\end{tabular}

Note: $\mathrm{LC}_{50}$ indicate the median lethal concentration; $\mathrm{SE}$ is the abbreviation of Standard error.

\subsection{Phloem Mobility of Conjugates}

Chlorfenapyr was selected as the control in the phloem systemicity test because tralopyril has an intolerable phytotoxicity to plants. Conjugates $\mathbf{4 2 - 4 5}$ were selected for further test because of their insecticidal activity. The phloem sap of conjugates $42-45$ or chlorfenapyr was collected within $2 \mathrm{~h}$ for detection. When the cotyledons were incubated in a chlorfenapyr solution at the same concentration, it was not detected in the phloem sap (Table 3). Upon observation, their concentrations in the phloem sap were approximately $24.84,41.88,36.77$, and $30.58 \mu \mathrm{M}$. The detected concentrations of conjugates $43-45$ were remarkably higher than that of conjugate 42 , and conjugate 43 had the best phloem mobility.

Table 3. Phloem mobility of $\mathbf{4 2 - 4 5}$ and chlorfenapyr. The cotyledons were incubated in a buffered solution containing $200 \mu \mathrm{M} \mathbf{4 2 - 4 5}$ and chlorfenapyr at pH 5.5. The sap was collected within two hour of incubation.

\begin{tabular}{cc}
\hline & Concentration in Phloem Sap $(\mu \mathrm{M})$ \\
\hline $\mathbf{4 2}$ & $24.8493 \pm 0.6730 \mathrm{~d}$ \\
$\mathbf{4 3}$ & $41.887 \pm 0.4591 \mathrm{a}$ \\
$\mathbf{4 4}$ & $36.7447 \pm 0.6832 \mathrm{~b}$ \\
$\mathbf{4 5}$ & $30.5837 \pm 1.9420 \mathrm{c}$ \\
chlorfenapyr & $0.00 \mathrm{e}$ \\
\hline
\end{tabular}

Note: English letters of a, b, c, d and e indicate the difference in $5 \%$ level.

The physicochemical properties of conjugates 42-45 were calculated to predict transmembrane behavior in plants (Table 4). The "Rule of Five" can be used to predict the diffusion of endogenous molecules or xenobiotics through plant membranes [24,25]. According to this rule, poor absorption, or permeation of small molecules is more probable when their MW, $\log \mathrm{P}, \mathrm{HBDs}$, and HBAs are more than $500 \mathrm{D}, 5$, 5, and 10, respectively; moreover, at least three in four parameters must satisfy this range [26]. Considering that the two parameters (MW and HBAs) of conjugates 42-45 in the table are out of range, 
they would be expected to diffuse through the membranes poorly. This result suggests the existence of a carrier-mediated absorption and transport mechanism other than passive diffusion, which is worthy of further study.

Table 4. Predicted physicochemical descriptor MW, $\log \mathrm{P}, \mathrm{pKa}, \mathrm{HBD}, \mathrm{HBA}$ of tralopyril, chlorfenapyr, $42,43,44$, and $45^{\text {a }}$.

\begin{tabular}{cccccc}
\hline Feature & MW (D) & logP & pKa & HBD & HBA \\
\hline Tralopyril & 349.53 & 4.02 & $14^{\mathrm{b}}$ & 1 & 2 \\
Chlorfenapyr & 407.61 & 4.76 & 14 & 0 & 3 \\
$\mathbf{4 2}$ & 633.81 & 3.00 & 3.91 & 3 & 11 \\
$\mathbf{4 3}$ & 647.83 & 3.22 & 3.92 & 3 & 11 \\
$\mathbf{4 4}$ & 661.86 & 3.52 & 3.92 & 3 & 11 \\
$\mathbf{4 5}$ & 675.88 & 3.76 & 3.92 & 3 & 11 \\
\hline
\end{tabular}

$\bar{a}$ All parameters were computed using ACD/Percepta 14.0.0 software. ${ }^{\mathrm{b}}$ Assumed nonacidic and assigned pKa of 14

Linker length is very important for the transporter recognition of carrier-medicated conjugates [8,27]. Based on the results of the phloem systemicity test, the linker arm with the three-atom unit was the optimal length for uptake and transport of glutamic acidtralopyril conjugates. Such an optimum in phloem mobility may be due to the structural requirement for the recognition by transporters. A shorter linker may lead to the interaction of amino acid moiety and tralopyril and affect the molecular recognition by transporters.

\subsection{Metabolism of Conjugates $\mathbf{4 2}$ and $\mathbf{4 3}$ in P. xylostella}

Conjugates $\mathbf{4 2}$ and $\mathbf{4 3}$ were selected to study the in vivo mechanism in P. xylostella and investigate the pro-insecticide properties of conjugates. Figure 2 shows that tralopyril was detected in all the P.xylostella treated with conjugates $\mathbf{4 2}$ and $\mathbf{4 3}$, and the tralopyril concentrations were 0.5950 and $0.3172 \mathrm{nmol} / \mathrm{kg}$, respectively, which is consistent with the experimental results of insecticidal activity (Table 2). The results suggest that the different linker lengths influenced the release of the active parent compound in P. xylostella. This behavior may involve the enzymatic bioactivation to release tralopyril because chlorfenapyr is released upon metabolism by P450s in vivo [28,29].

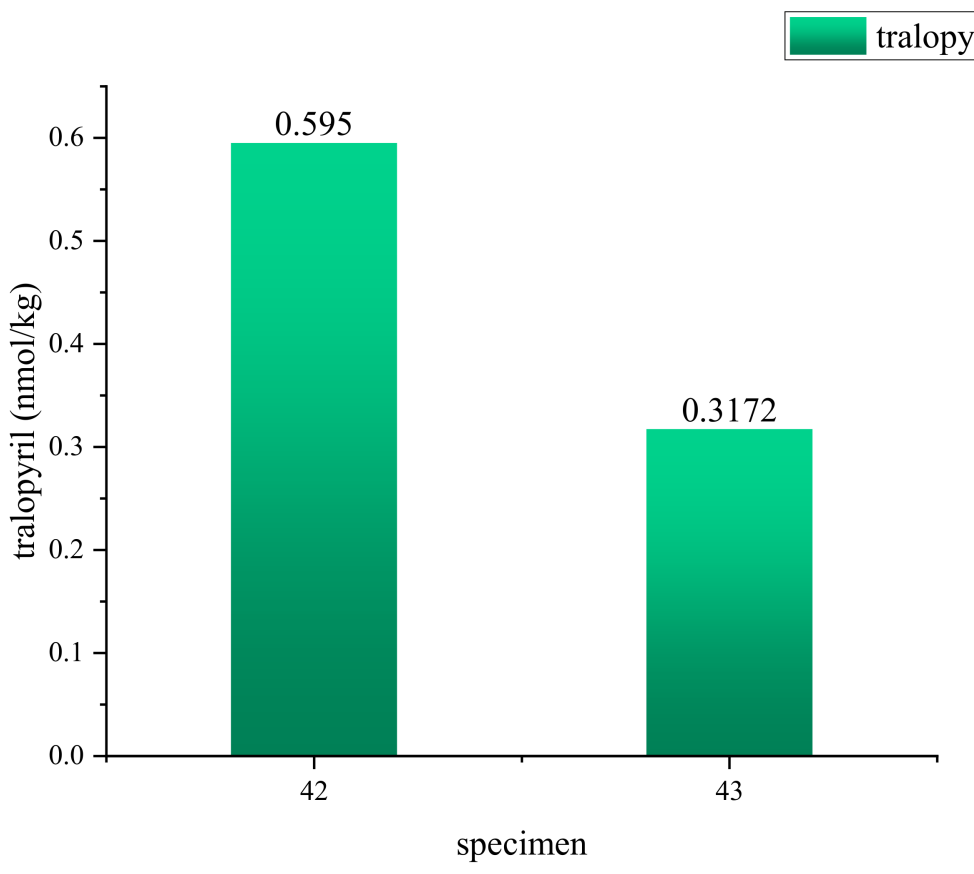

Figure 2. Metabolism of conjugates 42 and 43 in P. xylostella. 


\section{Conclusions}

In this study, nine conjugates containing tralopyril and glutamic acid were synthesized via click chemistry with good overall yield. The results concerning insecticidal activity against $P$. xylostella indicated that conjugates $42,43,44$, and 45 exhibited preferable activity under the tested concentrations. The metabolism of conjugates $\mathbf{4 2}$ and $\mathbf{4 3}$ in P. xylostella showed that they can release the active compound tralopyril in vivo. The results concerning phloem mobility demonstrated that the phloem mobilities of conjugates 43,44 , and 45 were higher than that of conjugate 42. Although the conjugates exhibited direct insecticidal activity that was lower than that of chlorfenapyr, conjugates $\mathbf{4 2}$ and $\mathbf{4 3}$ exhibited proinsecticide behavior. Such conjugates can achieve the primary transport of proinsecticide to the plant phloem. Then, the proinsecticide releases the active compound tralopyril at the desired site after being eaten by insects. The appropriate structural design of the linkers may enable controlling the bioactivation and phloem mobility of glutamic acid-tralopyril conjugates. This study expanded on the site-vectorized insecticide delivery strategy by investigating the effect of linker length on insecticidal activity and phloem mobility. As a promising phloem-mobile proinsecticide, the systemic insecticidal activity and phloem loading mechanism of conjugates $\mathbf{4 2}$ and $\mathbf{4 3}$ will be further investigatedin other plants.

Author Contributions: T.X.L., Y.C. and W.Y. conceived, designed andanalyzed the data; T.X.L., H.F.L. and C.Y.M. performed the experiments; Y.C. and W.Y. contributed reagents/materials/analysis tools; T.X.L. and W.Y. wrote the paper. All authors have read and agreed to the published version of the manuscript.

Funding: This work was supported by the National Natural Science Foundation of China (Grant No. 31760528) and the Independent Innovation Project of Guizhou academy of agricultural sciences (Grant No. [2014]005 and [2014]009).

Institutional Review Board Statement: Not applicable.

Informed Consent Statement: Not applicable.

Conflicts of Interest: The authors declare no conflict of interest.

\section{References}

1. Wu, H.X.; Xu, H.H.; Marivingt-Mounir, C.; Bonnemain, J.L.; Chollect, J.F. Vectorizing agrochemicals: Enhancing bioavailability via carrier-mediated transport. Pest Manag. Sci. 2019, 75, 1507-1516. [CrossRef] [PubMed]

2. Hsu, F.C.; Sun, K.; Kleier, D.A.; Fielding, M.J. Phloem mobility of xenobiotics VI. A phloem-mobile pro-nematicide based on oxamyl exhibiting root-specific activation in transgenic tobacco. Pestic. Sci. 1995, 44, 9-19. [CrossRef]

3. Brück, E.; Elbert, A.; Fischer, R.; Krueger, S.; Kühnhold, J.; Klueken, A.M.; Nauen, R.; Niebes, J.F.; Reckmann, U.; Schnorbach, H.J.; et al. Movento ${ }^{\circledR}$, an innovative ambimobile insecticide for sucking insect pest control in agriculture: Biological profile and field performance. Crop Prot. 2009, 28, 838-844. [CrossRef]

4. Jiang, D.X.; Lu, X.L.; Hu, S.; Zhang, X.B.; Xu, H.H. A new derivative of fipronil: Effect of adding a glycinyl group to the 5-amine of pyrazole on phloem mobility and insecticidal activity. Pestic. Biochem. Phys. 2009, 95, 126-130. [CrossRef]

5. Yang, W.; Wu, H.X.; Xu, H.H.; Hu, A.L.; Lu, M.L. Synthesis of Glucose-Fipronil conjugate and its phloem mobility. J. Agric. Food Chem. 2011, 59, 12534-12542. [CrossRef] [PubMed]

6. Müller, T.; Brancq, B.; Milius, A.; Okori, N.; Vaille, C.; Gauvrit, C. Ethoxylated rapeseed oil derivatives as novel adjuvants forherbicides. Pest Manag. Sci. 2002, 58, 1243-1249. [CrossRef]

7. Wu, H.X.; Marhadour, S.; Lei, Z.W.; Yang, W.; Marivingt-Mounir, C.; Bonnemain, J.L.; Chollet, J.F. Vectorization of agrochemicals: Amino acid carriers are more efficient than sugar carriers to translocate phenylpyrrole conjugates in the Ricinus system. Environ. Sci. Pollut. Res. 2018, 25, 14336-14349. [CrossRef]

8. Marhadour, S.; Wu, H.X.; Yang, W.; Marivingt-Mounir, C.; Bonnemain, J.L.; Chollet, J.F. Vectorisation of agrochemicals via amino acid carriers: Influence of the spacer arm structure on the phloem mobility of phenylpyrrole conjugates in the Ricinus system. Pest Manag. Sci. 2017, 73, 1972-1982. [CrossRef]

9. Mao, G.L.; Yan, Y.; Chen, Y.; Wang, B.F.; Xu, F.F.; Zhang, Z.X.; Lin, F.; Xu, H.H. Family of Ricinus communis monosaccharide transporters and RcSTP1 in promoting the uptake of a glucose-fipronil conjugate. J. Agric. Food Chem. 2017, 65, 6169-6178. [CrossRef]

10. Chen, Y.; Yan, Y.; Ren, Z.F.; Ganeteg, U.; Yao, G.K.; Li, Z.L.; Huang, T.; Li, J.H.; Tian, Y.Q.; Lin, F.; et al. AtLHT1 transporter can facilitate the uptake and translocation of a glycinergic-chlorantraniliprole conjugate in arabidopsis thaliana. J. Agric. Food Chem. 2018, 66, 12527-12535. [CrossRef] 
11. Oliveira, I.B.; Groh, K.J.; Stadnicka-Michalak, J.; Schönenberger, R.; Beiras, R.; Barroso, C.M.; Langford, K.H.; Thomas, K.V.; Suter, M.J.F. Tralopyril bioconcentration and effects on the gill proteome of the Mediterranean mussel Mytilus galloprovincialis. Aquat. Toxicol. 2016, 177, 198-210. [CrossRef] [PubMed]

12. Addor, R.W.; Furch, J.A.; Kuhn, D.G. Process for the Preparation of Insecticidal, Acaricidal and Nematicidal 2-Aryl-5(trifluoromethyl) Pyrrole Compounds. US 5030735,, 9 July 1991.

13. Raghavendra, K.; Barik, T.K.; Sharma, P.; Bhatt, R.M.; Srivastava, H.C.; Sreehari, U.; Dash, A.P. Chlorfenapyr: A new insecticide with novel mode of action can control pyrethroid resistant malaria vectors. Malar. J. 2011, 10, 397. [CrossRef] [PubMed]

14. Albers, P.H.; Klein, P.N.; Green, D.E.; Melancon, M.J.; Bradley, B.P.; Noguchi, G. Chlorfenapyr and mallard ducks: Overview, study design, macroscopic effects, and analytical chemistry. Environ. Toxicol. Chem. 2006, 25, 438-445. [CrossRef]

15. Chen, Y.; Lei, Z.W.; Zhang, Y.; Yang, W.; Liu, H.F.; Zhou, Y.F.; Yang, M.F. Influence of Pyranose and Spacer Arm Structures on Phloem Mobility and Insecticidal Activity of New Tralopyril Derivatives. Molecules 2017, 22, 1058. [CrossRef]

16. Yang, W.; Chen, Y.; Zhang, Y.; Gao, X.B.; Zhou, Y.F. Effects of introducing theanine or glutamic acid core to tralopyril on systemicity and insecticidal activity. Pestic. Biochem. Phys. 2016, 141, 29-40. [CrossRef]

17. We, Y.J.; Jiang, X.Y.; Yang, C.; Meng, H.Y.; Wang, B.F.; Wu, H.X.; Zhang, Z.X.; Xu, H.H. The linker length of glucose-fipronil conjugates has a major effect on the rate of bioactivation by $\beta$-glucosidase. Pest Manag. Sci. 2019, 75, $708-717$.

18. Lu, X.Q.; Bittman, R. Synthesis of a photoactivatable $(2 S, 3 R)$-sphingosyl phosphorylcholine analogue. J. Org. Chem. 2005, 70, 4746-4750. [CrossRef]

19. Chollet, J.F.; Rocher, F.; Jousse, C.; Deletage-Grandon, C.; Bashiardes, G.; Bonnemain, J.L. Synthesis and phloem mobility of acidic derivatives of the fungicide fenpiclonil. Pest Manag. Sci. 2004, 60, 1063-1072. [CrossRef] [PubMed]

20. Kallarackal, J.; Orlich, G.; Schobert, C.; Komor, E. Sucrose transport into the phloem of Ricinus communis L. seedlings as measured by the analysis of sieve-tube sap. Planta 1989, 177, 327-335. [CrossRef]

21. Wang, X.L.; Wang, J.; Cao, X.W.; Wang, F.L.; Yang, Y.H.; Wu, S.W.; Wu, Y.D. Long-term monitoring and characterization of resistance to chlorfenapyr in P. xylostella (Lepidoptera: Plutellidae) from China. Pest Manag. Sci. 2019, 75, 591-597. [CrossRef] [PubMed]

22. Gomes, A.; Dey, A.; Dasgupta, S.C. Black Tea (Camellia sinensis) Extract induced prenatal and postnatal toxicity in experimental albino rats. Pharmacogn. Mag. 2018, 13, S769-S774.

23. Hu, Z.D.; Feng, X.; Lin, Q.S.; Chen, H.Y.; Li, Z.U.; Yin, F.; Liang, P.; Gao, X.W. cDNA cloning and characterization of the carboxylesterase pxCCE016b from the diamondback moth, P. xylostella L. J. Integr Agric. 2016, 15, 1059-1068. [CrossRef]

24. Bhal, S.; Kassam, K.I.; Pearl, G. The rule of five revisited: Applying log D in place of log P in drug-likeness filters. Mol. Pharm. 2007, 4, 556-560. [CrossRef] [PubMed]

25. Rocher, F.; Chollet, J.F.; Legros, S.; Jousse, C.; Lemoine, R.; Faucher, M.; Bush, D.R.; Bonnemain, J.L. Salicylic acid transport in Ricinus communis involves a pH-dependent carrier system in addition to diffusion. Plant Physiol. 2009, 150, $2081-2091$. [CrossRef]

26. Lipinski, C.A.; Lombardo, F.; Dominy, B.W.; Feeney, P.J. Experimental and computational approaches to estimate solubility and permeability in drug discovery and development settings. Adv. Drug Deliv. Rev. 2012, 64, 4-17. [CrossRef]

27. Cecioni, S.; Praly, J.-P.; Matthews, S.E.; Wimmerová, M.; Imberty, A. Vidal, S. Rational design and synthesis of optimized glycoclusters for multivalent lectin-carbohydrate interactions: Influence of the linker arm. Chemistry 2012, 18, 6250-6263. [CrossRef]

28. Jeschke, P. Propesticides and their use as agrochemicals. Pest Manag. Sci. 2016, 72, 210-225. [CrossRef] [PubMed]

29. Zhao, Y.; Mao, C.H.; Li, Y.Q.; Zhang, P.X..; Huang, Z.Q.; Bi, F.C.; Huang, R.Q.; Wang, Q.M. Synthesis, crystal structure, and insecticidal activity of novel N-alkyloxyoxalyl derivatives of 2-arylpyrrole. J. Agric. Food Chem. 2008, 56, 7326-7332. [CrossRef] [PubMed] 